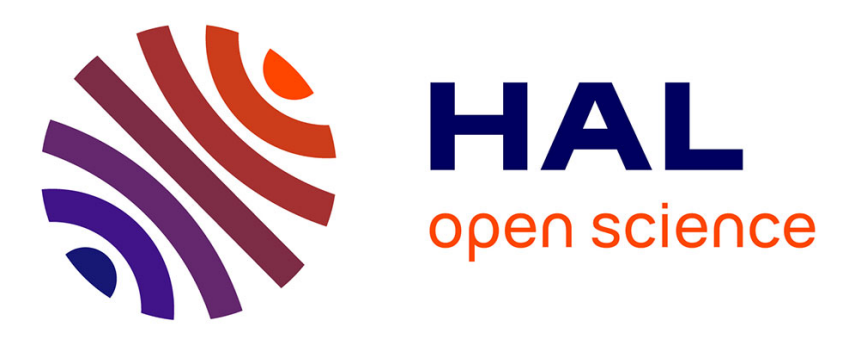

\title{
Low complex sensor-based shared control for power wheelchair navigation
}

Louise Devigne, Vishnu Karakkat Narayanan, François Pasteau, Marie Babel

\section{To cite this version:}

Louise Devigne, Vishnu Karakkat Narayanan, François Pasteau, Marie Babel. Low complex sensorbased shared control for power wheelchair navigation. IROS2016 - IEEE/RSJ International Conference on Intelligent Robots and Systems, Oct 2016, Daejeon, South Korea. pp.5434-5439. hal-01355410v2

\section{HAL Id: hal-01355410 \\ https://hal.inria.fr/hal-01355410v2}

Submitted on 28 Nov 2016

HAL is a multi-disciplinary open access archive for the deposit and dissemination of scientific research documents, whether they are published or not. The documents may come from teaching and research institutions in France or abroad, or from public or private research centers.
L'archive ouverte pluridisciplinaire HAL, est destinée au dépôt et à la diffusion de documents scientifiques de niveau recherche, publiés ou non, émanant des établissements d'enseignement et de recherche français ou étrangers, des laboratoires publics ou privés. 


\title{
Low complex sensor-based shared control for power wheelchair navigation
}

\author{
Louise Devigne $^{1}$, Vishnu K.Narayanan ${ }^{2}$, \\ François Pasteau ${ }^{4}$, Marie Babel $^{3}$
}

\begin{abstract}
Motor or visual impairments may prevent a user from steering a wheelchair effectively in indoor environments. In such cases, joystick jerks arising from uncontrolled motions may lead to collisions with obstacles. We here propose a perceptive shared control system that progressively corrects the trajectory as a user manually drives the wheelchair, by means of a sensor-based shared control law capable of smoothly avoiding obstacles. This control law is based on a low complex optimization framework validated through simulations and extensive clinical trials. The provided model uses distance information. Therefore, for low-cost considerations, we use ultrasonic sensors to measure the distances around the wheelchair. The solution therefore provides an efficient assistive tool that does not alter the quality of experience perceived by the user, while ensuring his security in hazardous situations.
\end{abstract}

\section{INTRODUCTION}

In the world, more than 200 million people experience major mobility difficulties because of their impairments [1]. Defining and understanding the disability (including global ageing) [2] constitute the first step in envisaging responses towards disabilities.

Recent studies dealing with the development of Assistive Technologies (AT) highlight the importance of enhancing user autonomy and social inclusion [3]. In particular, a reduced mobility can deeply affect the capacity of a human being in remaining socially active. Achieving autonomy thus requires dedicated smart technologies that can contribute to the well-being as well as the personal dignity [1]. In this context, people with motor impairments who may also have cognitive and/or visual impairments can be prescribed a power wheelchair after a rigorous evaluation of their ability to drive safely [4][5].

Indeed, maneuvering a power wheelchair requires dedicated mobility skills as well as cognitive and visualperceptual abilities [6]. Even though these mobility skills can typically be obtained after a training period [7], some individuals still remain unauthorized to use a power wheelchair because of cognitive disorders, even with adapted interfaces [8]. This situation unfortunately leads to a loss of autonomy and independence, and contributes to social isolation. Then,

\footnotetext{
*This work was not supported by any organization

${ }^{1}$ Louise Devigne is with the Rehabilitation Center Pôle Saint Hélier, the Lagadic Team at IRISA/Inria Rennes and INSA Rennes, France

${ }^{2}$ Vishnu K.Narayanan is with the Lagadic Team at IRISA/Inria Rennes, France

${ }^{3}$ Marie Babel and François Pasteau is with the Lagadic Team at IRISA/Inria Rennes and INSA Rennes, France marie.babeleirisa.fr

${ }^{4}$ François Pasteau is with the Ergovie Company
}

the design of an efficient robotic driving assistance is clearly expected.

In this context, many recent research projects have focused on the study of Smart Wheelchair issues. Partial or fully assistive systems were designed within the NavChair [9], Radhar [10], SYSIASS [11] and COALAS projects [12] in order to propose safe navigation assistance. However, the main bottleneck of such solutions remains the final cost of the assisted navigation system, as well as the efficiency and the robustness [13].

We aim at designing a collision avoidance system matching both assistance and economic issues. For a widespread usage, it should meet the following characteristics:

- a low-cost embedded Plug and Play system that is immediately compliant with commercialized wheelchairs to guarantee an easy setup and maintenance,

- an efficient and robust collision avoidance framework which preserves the navigation comfort for a good quality of experience,

- a power efficient platform that does not reduce the battery efficiency, while keeping the wheelchair form factor (typically important in case of narrow spaces or for user transfers).

The related robotic control framework follows a shared control strategy. In the literature, three different strategies have been identified [14][15]. The first one is based on a goal prediction framework, and requires odometry and usually expensive sensors such as LIDARs. The second one is called behavior-based shared control and switches alternatively between different modes of navigation leading to potentially unwanted discontinuities in the control law. The third solution, denoted as continuous shared control approach, regulates the assistance in a progressive manner while fusing the user control with a collision avoidance framework.

In this paper, the proposed solution follows the continuous shared control strategy requirements. Among these control strategies, Dynamic Window Approaches are one of the most used solutions. Originally designed for reactive autonomous navigation purposes [16], the control law is directly computed in the space of velocities where forbidden and allowed areas are defined. Resulting velocities applied to the robot are obtained by maximizing an objective function. In [17], a Shared Control Dynamic Window Approach has been detailed but only applied to simulated environments. In our work, the proposed shared control solution is inspired from the Dynamic Window Approaches. We use an adapted 
representation of the search space allowing a geometric minimization of the cost function. However, contrary to [17] which is a pose-based servoing process, our solution is sensor-based thus also lowering the complexity of the control law.

In [18], we have presented the HandiViz project and the robotic system used for clinical trials. Here, although the shared control method relies on the same principles, we generalize the notations and detail the cost function minimization algorithm. In addition, the model is validated through simulations.

The next Section outlines the shared control approach for realizing a smooth trajectory correction. Section III presents simulations of the system, thus highlighting the behavior of the servoing process. Section IV presents the clinical trials which were conducted within the Rehabilitation Center of Pôle Saint Hélier in Rennes (France), where 23 volunteers have participated in these trials.

\section{GENERIC SHARED CONTROL APPROACH}

To achieve a robust obstacle avoidance solution, the proposed algorithm fuses the manual control coming from a traditional joystick with a set of constraints deduced from the sensors. As with the Dynamic Window Approach, this shared control law is based on the definition of two distinct areas (allowed/forbidden areas) in the wheelchair velocity domain. However, the proposed method uses a sensor-based servoing approach to provide a low complex algorithm and to simplify the geometry of the areas. This section explains how the constraints are computed from the sensors and how the allowed and forbidden areas are defined.

\section{A. Modelling}

As shown on Fig. 1,

- let $\mathbf{u}=(u, \omega)$ be the velocity of the wheelchair,

- let $\mathbf{v}_{c_{i}}=\left(u_{c_{i}}, \omega_{c_{i}}\right)$ be the velocity of the sensor $c_{i}$,

- let $x_{i}$ be the distance from the sensor $c_{i}$ to the obstacle,

- let $x_{i}^{*}$ be a minimum allowed distance from the sensor $c_{i}$ to the obstacle,

- let $e_{c_{i}}=x_{i}-x_{i}^{*}$ be the error between $x_{i}$ and $x_{i}^{*}$.
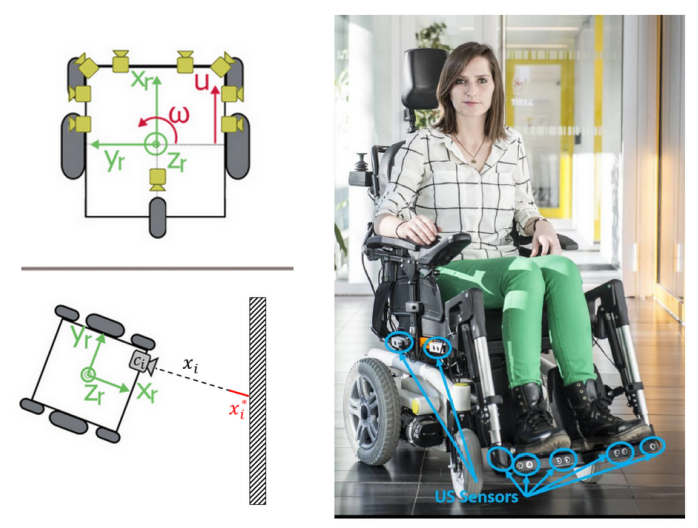

Fig. 1: Definition of the robot frame
We can define a Jacobian $\mathbf{J}$ and the interaction matrix $\mathbf{L}_{x_{i}}$ for each sensor $c_{i}$ such that

$$
\begin{aligned}
\mathbf{v}_{c_{i}} & =\mathbf{J} \mathbf{u} \\
\dot{x}_{i} & =\mathbf{L}_{x_{i}} \mathbf{v}_{c_{i}} .
\end{aligned}
$$

By combining equations (1) and (2), we obtain

$$
\dot{x_{i}}=\mathbf{L}_{x_{i}} \mathbf{J} \mathbf{u} \text {. }
$$

To avoid collision with any static or dynamic obstacle, we constrain $\dot{x}_{i}$ by a minimum value $-\lambda e_{c_{i}}$ leading to the design of a proportional corrector. Hence, we get

$$
\dot{x}_{i} \geq-\lambda e_{c_{i}}
$$

By combining equation (3) and the inequality (4), we get

$$
\mathbf{L}_{x_{i}} \mathbf{J} \mathbf{u} \geq-\lambda e_{c_{i}}
$$

Inequality (5) can also be rewritten as

$$
\mathbf{A}_{i} \mathbf{u} \geq B_{i}
$$

with $\mathbf{A}_{i}=\mathbf{L}_{x_{i}} \mathbf{J}$ and $B_{i}=-\lambda e_{c_{i}}$. This defines a half-plane in the $u-\omega$ plane as shown on Fig. 2 .

When considering $\mathrm{N}$ sensors, we can rewrite inequality (6) as

$$
\mathbf{A} \mathbf{u} \geq \mathbf{B}
$$

using $\mathbf{A}=\left[\begin{array}{c}\mathbf{A}_{0} \\ \vdots \\ \mathbf{A}_{N-1}\end{array}\right]$ and $\mathbf{B}=\left[\begin{array}{c}B_{0} \\ \vdots \\ B_{N-1}\end{array}\right]$.

\section{B. Computing control values}

- Let $\mathbf{u}_{o p}=\left(u_{o p}, \omega_{o p}\right)$ be the user desired velocity,

- let $\mathbf{u}_{c m d}=\left(u_{c m d}, \omega_{c m d}\right)$ be the robot velocity.

$\mathbf{u}_{c m d}$ is computed from $\mathbf{u}_{o p}$ under constraints (7) by minimizing a cost function $f$. This can be written as an optimization problem using

$$
\left\{\begin{aligned}
\mathbf{u}_{c m d} & =\min _{\mathbf{u}} f\left(\mathbf{u}_{o p}, \mathbf{u}\right) \\
\mathbf{A} \mathbf{u} & \geq \mathbf{B}
\end{aligned}\right.
$$

We define the function $f$ such that

$$
f\left(\mathbf{u}_{o p}, \mathbf{u}\right)=\alpha\left(u-u_{o p}\right)^{2}+\beta\left(\omega-\omega_{o p}\right)^{2}
$$

with $\alpha \geq 0$ and $\beta \geq 0$. In our practical setup, $\alpha=\left|u_{o p}\right|$ and $\beta=\left|\omega_{o p}\right|$. This provides a control value $\mathbf{u}_{c m d}$ which respects as much as possible the main direction given by the user. We performed tests with regular users to define our parameter values.

Then the problem can be written as a classical quadratic optimization problem using

$$
\left\{\begin{aligned}
\mathbf{u}_{c m d} & =\min _{\mathbf{u}} \mathbf{g}^{T} \mathbf{u}+\frac{1}{2} \mathbf{u}^{T} \mathbf{H u} \\
\mathbf{A u} & \geq \mathbf{B}
\end{aligned}\right.
$$

with $\mathbf{g}=\mathbf{u}_{o p}{ }^{T}\left[\begin{array}{cc}-\alpha & 0 \\ 0 & -\beta\end{array}\right]$ and $\mathbf{H}=\left[\begin{array}{cc}\alpha & 0 \\ 0 & \beta\end{array}\right]$. 


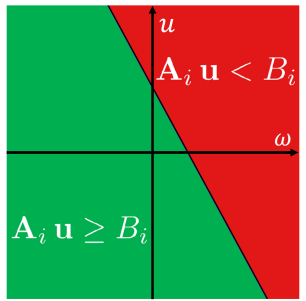

Fig. 2: Half plane in the $u-\omega$ plane

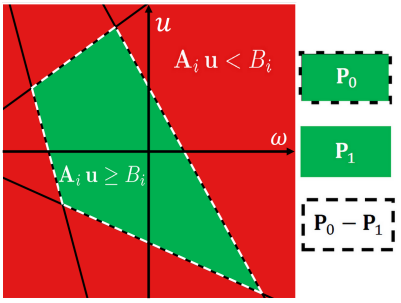

Fig. 3: Definition of $\mathbf{P}_{0}$ and $\mathbf{P}_{1}$

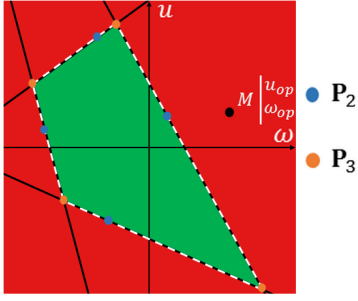

Fig. 4: Definition of $\mathbf{P}_{2}$ and $\mathbf{P}_{3}$
As shown on Fig. 3, we can define two sets $\mathbf{P}_{0}$ and $\mathbf{P}_{1}$ such that

$$
\begin{aligned}
& \mathbf{P}_{0}=\{\mathbf{u} \mid \mathbf{A} \mathbf{u} \geq \mathbf{B}\} \\
& \mathbf{P}_{1}=\{\mathbf{u} \mid \mathbf{A} \mathbf{u}>\mathbf{B}\} .
\end{aligned}
$$

Hence the problem (10) can be resolved only if $\mathbf{P}_{0} \neq \emptyset$. If $\mathbf{P}_{0}=\emptyset, x_{i}^{*}$ can be dynamically decreased to expand the size of $\mathbf{P}_{0}$, thus allowing the resolution of the problem.

In fact, the quadratic optimization problem (10) can be solved by distinguishing two different cases:

- Case 1: if $\mathbf{u}_{o p} \in \mathbf{P}_{0}$, then $\mathbf{u}_{c m d}=\mathbf{u}_{o p}$ (no collision can occur),

- Case 2: otherwise, as the cost function is convex and its minimum is reached when $\mathbf{u}=\mathbf{u}_{o p}, \mathbf{u}_{c m d} \in \mathbf{P}_{0}-\mathbf{P}_{1}$ meaning that there exists at least one $i$ such that $\mathbf{A}_{i} \mathbf{u}=B_{i}$.

\section{Cost function minimization}

Under Case 2, as the velocity domain is a 2D plan, only 2 different scenarios can occur:

- Case 2.1: there exists one and only one $i$ such that $\mathbf{A}_{i} \mathbf{u}=B_{i}$,

- Case 2.2: there exists one and only one couple $\{i, j\}$ such that $\left(\mathbf{A}_{i} \mathbf{u}=B_{i}, \mathbf{A}_{j} \mathbf{u}=B_{j}\right)$.

Under Case 2.1, there exists one and only one $i$ such that $\mathbf{A}_{i} \mathbf{u}=B_{i}$. For each sensor $c_{i}$, the system

$$
\left\{\begin{aligned}
\hat{\mathbf{u}} & =\min _{\mathbf{u}} \mathbf{g}^{T} \mathbf{u}+\frac{1}{2} \mathbf{u}^{T} \mathbf{H u} \\
\mathbf{A}_{i} \mathbf{u} & =B_{i}
\end{aligned}\right.
$$

has to be solved. We then look for a set $\mathbf{P}_{2}=\left\{\hat{\mathbf{u}} \mid \hat{\mathbf{u}} \in \mathbf{P}_{0}\right\}$ of possible solutions (Fig. 4). The system (12) corresponds to a cost function minimization along a line and is easily solved. As all the constraints are linear, we get $\operatorname{card}\left(\mathbf{P}_{2}\right) \leq N$. This case corresponds to the minimum being on a segment of the polygon described by $\mathbf{P}_{0}$.

Under Case 2.2, there exists one and only one couple $\{i, j\}$ such that $\left(\mathbf{A}_{i} \mathbf{u}=B_{i}, \mathbf{A}_{j} \mathbf{u}=B_{j}\right)$. For each couple of sensors $\left\{c_{i}, c_{j}\right\}$, the system

$$
\left\{\begin{array}{l}
\mathbf{A}_{i} \hat{\mathbf{u}}=B_{i} \\
\mathbf{A}_{j} \hat{\mathbf{u}}=B_{j}
\end{array}\right.
$$

is solved. We then look for a set $\mathbf{P}_{3}=\left\{\hat{\mathbf{u}} \mid \hat{\mathbf{u}} \in \mathbf{P}_{0}\right\}$ of possible solutions (Fig. 4). The system (13) corresponds to a linear system and is once again easily solved. As all the constraints are linear, we also get $\operatorname{card}\left(\mathbf{P}_{3}\right) \leq N$. This case corresponds to the minimum being on a corner of the polygon described by $\mathbf{P}_{0}$.

Finally, the optimization problem (10) can be written as

$$
\left\{\mathbf{u}_{c m d}=\min _{\mathbf{u} \in \mathbf{P}_{2} \cup \mathbf{P}_{3}} \mathbf{g}^{T} \mathbf{u}+\frac{1}{2} \mathbf{u}^{T} \mathbf{H u}\right.
$$

An exhaustive search on $\mathbf{P}_{2} \cup \mathbf{P}_{3}$ leads to at most $2 N$ computations of the cost function and provides the solution of the problem (10).

To sum up, the proposed shared control solution allows the user to control the wheelchair while observing safety constraints. The formulation of the problem leads to solve a simple quadratic system under constraints. If it exhibits similarities with the Dynamic Window Approach (i.e. forbidden/allowed areas), it provides a low complex method using a sensor-based servoing approach. This model also does not rely on a specific type of sensors. Moreover, there is no need of fine calibration and no limit in the number $\mathrm{N}$ of sensors that can be used.

\section{Simulations}

In this section, we present results of a simulation to better demonstrate the properties of the proposed shared control law. As shown on Fig. 6a, the virtual environment corresponds to a 2 metre wide and 10 metre long corridor with 4 obstacles. The virtual wheelchair starts from the beginning of the corridor facing the top wall with an angle of $60^{\circ}$. The user input is constant and equal to $100 \%$ of the maximum speed of the wheelchair. The wheelchair is equipped with 9 virtual sensors with a refresh rate of $50 \mathrm{~Hz}$. This number of sensors has been chosen as a worst case scenario to test the collision avoidance all around the wheelchair. The dynamics of the wheelchair are simulated using values corresponding to the average parametrization of a real-user wheelchair:

- a maximum forward speed of $0.9 \mathrm{~m} . \mathrm{s}^{-1}$,

- a maximum forward acceleration of $2 \mathrm{~m} . \mathrm{s}^{-2}$,

- a maximum rotational speed of 1 rad.s $s^{-1}$,

- a maximum rotational acceleration of 3 rad.s $s^{-2}$.

Fig. 5 shows the whole algorithm used for simulation purposes. Distances to obstacles $x_{i}$ are computed from the map of the environment and the pose of the virtual wheelchair. Constraints are estimated using the Inequality (7). From the user joystick position, the desired speed $\mathbf{u}_{o p}$ of the wheelchair is estimated. From $\mathbf{u}_{o p}$ using Equation (14), the quadratic minimization is performed leading to the velocity $\mathbf{u}_{c m d}$ which is then applied to the wheelchair. 


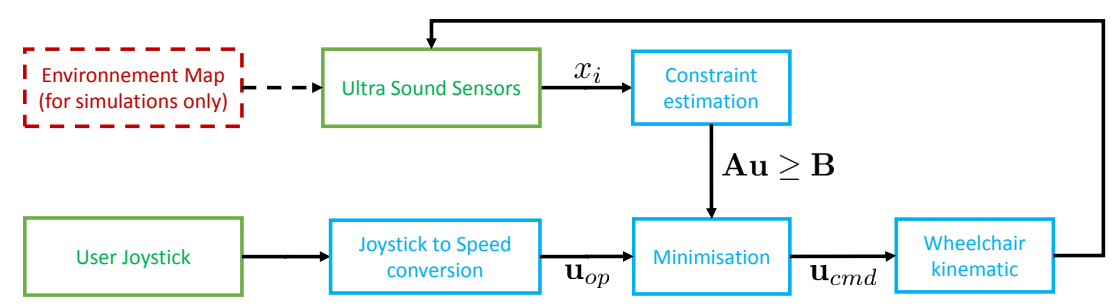

Fig. 5: Simulated algorithm

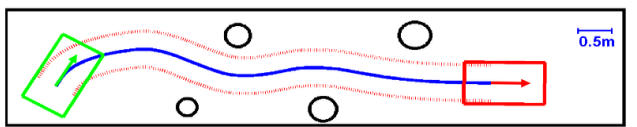

(a) Simulated trajectory for the wheelchair

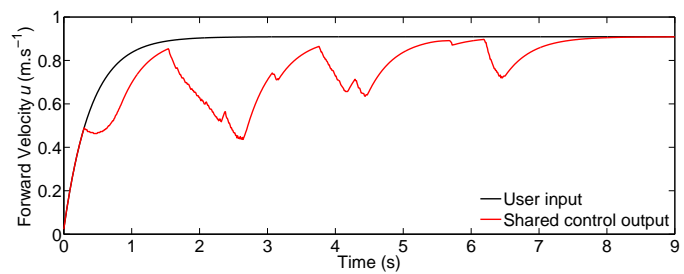

(b) Forward velocity applied to the wheelchair

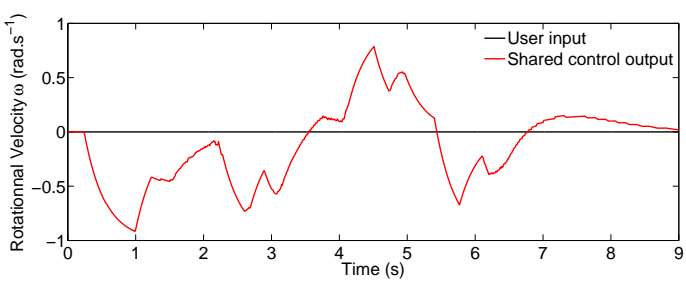

(c) Rotational velocity applied to the wheelchair

Fig. 6: Simulation results: (b) and (c) represent the corrected forward velocity and rotational velocity with respect to the timeline of the obstacle avoidance represented in (a).

As the proposed algorithm does not rely on a map to compute the shared control law, it is to be noted that the map and the pose are used for simulation visualization purposes only.

Fig. 6a shows the trajectory of the wheelchair during the simulation. Without any map of the environment and without any correction from the user (only a constant forward velocity is applied), the wheelchair moves smoothly around the obstacles and follows the corridor.

As the simulated user joystick is set to input a constant forward velocity, the cost function expressed by Equation (9) used in the simulation tries to maximize the forward velocity. Therefore, as shown on Fig. 7, the chosen couple of forward and rotational velocities is the one that maximizes the forward velocity and not the one that is the closest in the sense of the Euclidean norm to the user input. As shown on Fig. 6b, when getting closer to an obstacle, the wheelchair

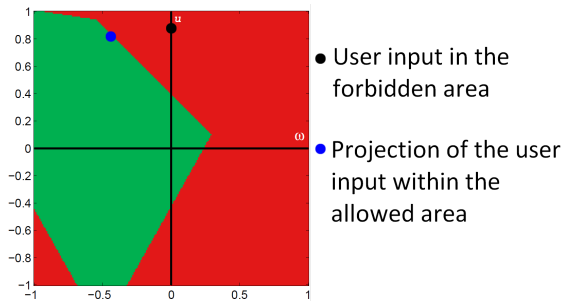

Fig. 7: Example of allowed/forbidden areas during the simulation

slows down to provide a comfortable and safe motion.

With a constant forward velocity input, the rotational velocity is also servoed to avoid obstacles (Fig. 6c). As the user only asks to move forward, the direction chosen by the control law depends on the orientation of the wheelchair with regards to the corridor and the obstacles.

We have then demonstrated the ability of the system to provide a smooth trajectory correction while avoiding obstacles. In particular, the simulations validate the case when the user input is kept constant.

\section{Clinical trials}

Validating an assistive solution such as the proposed shared control solution requires clinical validation. Clinical trials are a key step to measure its social benefit as well as acceptability and suitability to match user expectations. In this section, we detail the related methodology and highlight the performances of the embedded robotic system.

This clinical trial has formerly received a favorable opinion from the local ethics committee of Pontchaillou Hospital (Rennes, France).

\section{A. Experimental setup}

An off-the-shelf power wheelchair from the You-Q company, more specifically the Luca model, was used to conduct the clinical trials. Such a wheelchair is equipped with 5 wheels, two of which are actuated and the three others act as caster wheels. To operate the wheelchair, the user controls a standard joystick coming from Penny \& Giles. To perform the collision avoidance algorithm and provide a low cost and widespread solution, 9 low cost ultrasonic sensors are installed on the wheelchair according to Fig. 1. This setup corresponds to the one used during simulations. Those 9 ultrasonic sensors give measurement with a refresh rate of $50 \mathrm{~Hz}$. The algorithm itself runs on an embedded Quad 
Core $900 \mathrm{MHz}$ CPU board. As the algorithm is low complex, the CPU load on such an ARM board is under 5\%. All calculations can be performed during one cycle of the CAN bus leading to a latency of $10 \mathrm{~ms}$ which has been shown to be undetectable by users. Finally, to activate the algorithm and to record the experiments, a user interface designed for the clinicians only is installed behind the wheelchair. With this setup, the users are not aware of the activation/deactivation of the collision avoidance solution.

\section{B. Clinical trials: methodology}

The clinical trials were conducted within the Rehabilitation Center Pôle Saint Hélier (Rennes, France). To validate the acceptability and to analyze the performances of the proposed driving assistance, 23 wheelchair users volunteered to participate to this study. These volunteers had different neurological pathologies inducing motor and possibly cognitive and/or visual impairments including: tetraplegia, cerebral palsy, amputations, brain injury, stroke, etc. A single configuration of the system was proposed to ensure that users perform in the same conditions.

An ecological circuit proposed by the medical staff was defined with static and dynamic obstacles (Fig. 8). These tests took place in the principal hallway of the rehabilitation center during working hours; therefore patients, carers and other wheelchair users were circulating as usual during the tests. Volunteers had to realize difficult maneuvers in narrow spaces (turning around, rolling back) in order to simulate environments that are typically encountered at home. They receive no further indications except from global indications concerning the circuit: the trajectory was not imposed.

The evaluation methodology required random doubleblind tests. Each volunteer navigated twice, with or without activation of the driving assistance. At the end of each trial, a QUEST-like questionnaire was proposed [19].

\section{Results}

Fig. 9 shows the number of collisions for each experiment. Without assistance, 11 out of the 23 participants collided a total of 20 times whereas with assistance only 5 hit a total number of 10 obstacles. Of those 10 collisions, 6 of them were during backward motion where the only installed sensor was not enough to detect obstacles, 2 of them where the collisions occurred with a foot of the user and finally 2 where the sensors did not detect the obstacle soon enough to avoid the collision.

As shown on Fig. 10, QUEST scores between tests with and without assistance remain very close with slightly better results for the assisted experiments. This shows the acceptability of the solution from the user point of view.

Fig. 11 shows the duration of the experiments. On average, experiments with assistance last $13 \mathrm{~s}$ more than without, meaning a $5.5 \%$ increase in time. The biggest difference is 93s.

As shown on Fig. 12, the algorithm is actually correcting the trajectory between $2 \%$ and $35 \%$ of the total time of the experiments. There is a strong discrepancy between the scores of the first and second half of the participants. Indeed, even if participants in the first half were considered as experts in driving, they were also the ones presenting more severe forms of cognitive or visual impairments, thus activating the collision avoidance strategy more often.

\section{CONCLUSion}

This paper illustrated a low-cost system for providing robotic assistance when a user drives a power wheelchair manually. Even if tests were performed with the same configuration for all users, it is possible to modify system parameters such as security margins, speed and the number of sensors: the system is adaptive and can be configured to match the needs of every user. User output is blended with sensor-based constraints in order to devise an intuitive shared control scheme that is capable of assisting the user progressively when needed. The behavior of the system in simulation as well as in practice demonstrates the effectiveness of the proposed control scheme for assisted indoor wheelchair navigation. The shared control model presented here is shown to be flexible, low complex, independent of the sensor type and can be adapted easily for commercial/research purposes. The trials with regular users identified the remaining shortcomings of our system in terms of sensor positioning. It also showed the acceptability of the system from the user point of view. Future works aim at testing the solution outdoors as well as testing the system with volunteers over a long period of time at home.

\section{ACKNOWLEDGEMENT}

The authors would like to thank Éric Bazin and Sylvain Guégan from INSA Rennes, Amélie Colin and Sophie Achille-Fauveau from Healthcare Network Breizh PC and Philippe Gallien, Benoît Nicolas and Bastien Fraudet from the Rehabilitation Center Pôle Saint Hélier. Also, the authors would like to thank all the volunteers who contributed to this study.

\section{REFERENCES}

[1] M. del Carmen Malbrán, "World Report on Disability," Journal of Policy and Practice in Intellectual Disabilities, vol. 8, no. 4, pp. 290290, Dec. 2011

[2] J. Beard, S. Biggs, D. E. Bloom, L. P. Fried, P. R. Hogan, A. Kalache, and S. J. Olshansky, "Global Population Ageing: Peril or Promise?" Program on the Global Demography of Aging, Tech. Rep. 8912, Jan. 2012

[3] A. Helal, M. Mokhtari, and B. Abdulrazak, The engineering handbook of smart technology for aging, disability, and independence. Wiley, 2008

[4] S. Lukersmith, L. Radbron, and K. Hopman, "Development of clinical guidelines for the prescription of a seated wheelchair or mobility scooter for people with traumatic brain injury or spinal cord injury," Australian Occupational Therapy Journal, vol. 60, no. 6, pp. 378-386, 2013.

[5] F. Routhier, C. Vincent, J. Desrosiers, and S. Nadeau, "Mobility of wheelchair users: a proposed performance assessment framework," Disability \& Rehabilitation, vol. 25, no. 1, pp. 19-34, 2003.

[6] R. H. L. Wang, "Enabling power wheelchair mobility with long-term care home residents with cognitive impairments," Ph.D. dissertation, University of Toronto, 2011.

[7] A. M. Cook and J. M. Polgar, Assistive technologies: Principles and practice. Elsevier Health Sciences, 2014. 


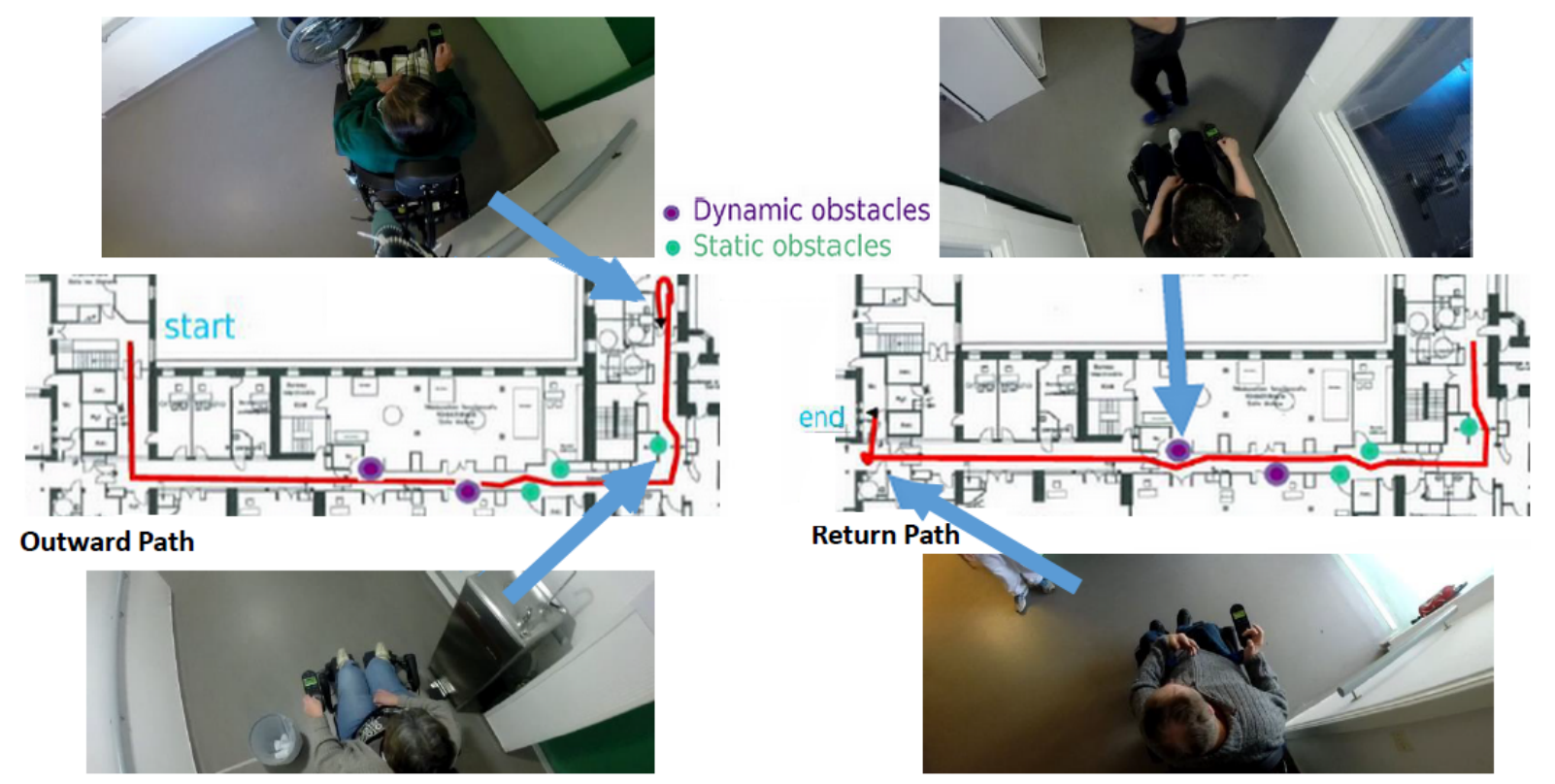

Fig. 8: Map of the experiments with key maneuvers

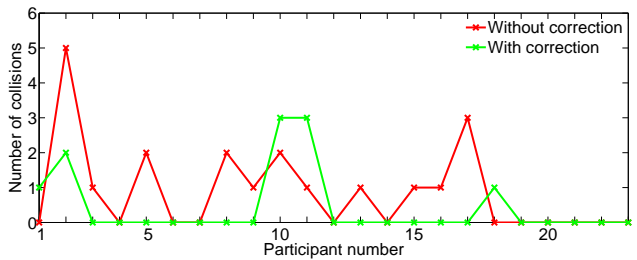

Fig. 9: Number of collisions

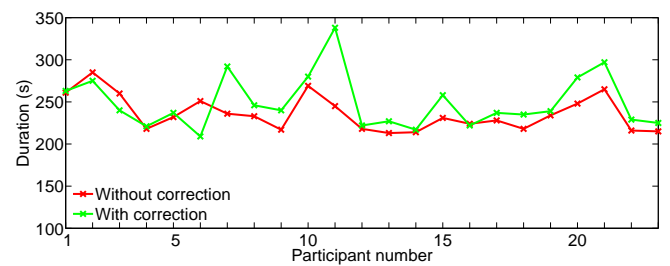

Fig. 11: Durations in $\mathrm{s}$

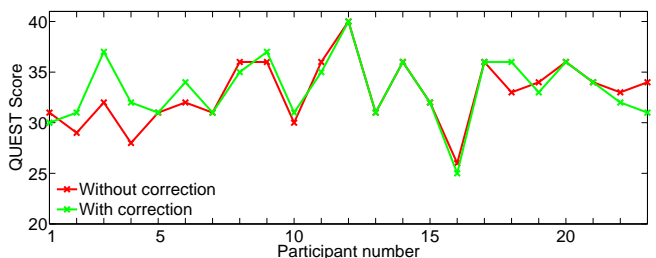

Fig. 10: QUEST scores

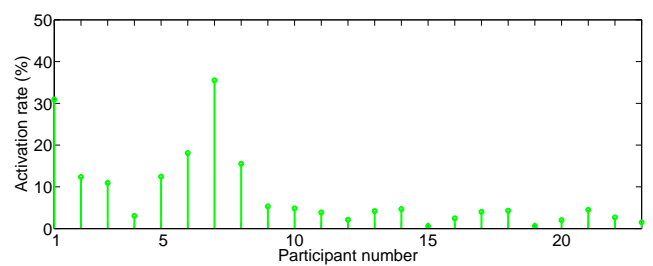

Fig. 12: Activation rates in (\%)
[8] L. Fehr, W. E. Langbein, and S. B. Skaar, "Adequacy of power wheelchair control interfaces for persons with severe disabilities: A clinical survey," Journal of rehabilitation research and development, vol. 37, no. 3, pp. 353-360, 2000.

[9] S. P. Levine, D. A. Bell, L. A. Jaros, R. C. Simpson, Y. Koren, and J. Borenstein, "The NavChair Assistive Wheelchair Navigation System," IEEE Transactions on Rehabilitation Engineering, vol. 7, pp. 443-451, 1999.

[10] E. Demeester, E. EB Vander Poorten, A. Hüntemann, and J. De Schutter, "Wheelchair Navigation Assistance in the FP7 Project RADHAR: Objectives and Current State," in Proc. of 1st International Conference on Systems and Computer Science (ICSCS), 2012.

[11] A. Kokosy, T. Floquet, G. Howells, H. Hu, M. Pepper, and C. Donz, "SYSIASS An Intelligent Powered Wheelchair," in Proc. of International Conference on Systems and Computer Science, 2012.

[12] R. Li, L. Wei, D. Gu, H. Hu, and K. McDonald-Maier, "Multi-layered map based navigation and interaction for an intelligent wheelchair," in Proc. of IEEE International Conference on Robotics and Biomimetics (ROBIO), Dec 2013, pp. 115-120.

[13] R. C. Simpson, "Smart wheelchairs: A literature review." Journal of rehabilitation research and development, vol. 42, no. 4, pp. 423-436, 2004.

[14] R. Cooper, "Intelligent control of power wheelchairs," IEEE Engineer- ing in Medicine and Biology Magazine, vol. 14, no. 4, pp. 423-431, Jul. 1995.

[15] C. Urdiales, J. M. Peula, M. Fdez-Carmona, C. Barru, E. J. Prez, I. Snchez-Tato, J. C. d. Toro, F. Galluppi, U. Corts, R. Annichiaricco, C. Caltagirone, and F. Sandoval, "A new multi-criteria optimization strategy for shared control in wheelchair assisted navigation," $A u$ tonomous Robots, vol. 30, no. 2, pp. 179-197, Oct. 2010.

[16] D. Fox, W. Burgard, and S. Thrun, "The dynamic window approach to collision avoidance," IEEE Robotics Automation Magazine, vol. 4, no. 1, pp. 23-33, Mar. 1997.

[17] P. Inigo-Blasco, F. Diaz-del Rio, S. Vicente Diaz, and D. Cagigas Muniz, "The Shared Control Dynamic Window Approach for Non-Holonomic Semi-Autonomous Robots," in Proc. of International Symposium on Robotics, Jun. 2014, pp. 1-6.

[18] M. Babel, F. Pasteau, S. Guégan, P. Gallien, B. Nicolas, B. Fraudet, S. Achille-Fauveau, and D. Guillard, "HandiViz project: clinical validation of a driving assistance for electrical wheelchair," in IEEE Workshop On Advanced Robotics And Its Social Impacts (ARSO), Lyon, France, Jul. 2015.

[19] L. Demers, R. Weiss-Lambrou, and B. Ska, "The quebec user evaluation of satisfaction with assistive technology (quest 2.0): an overview and recent progress," Technology and Disability, vol. 14, no. 3, pp. 101-105, 2002. 